Pacific Journal of Mathematic 


\section{SINGULAR INTEGRALS AND POSITIVE KERNELS}

\section{R. Putnam}

Let $k(x, t)$ be a $C^{1}$ kernel of a positive integral operator on $L^{2}(E)$ where $E$ is compact. It is shown that certain singular self-adjoint operators $A$ on $L^{2}(E)$, consisting of the sum of a multiplication operator and a generalized Hilbert transform integral operator with Kernel $i k(x, t)(x-t)^{-1}$, are analogous to-sometimes even to the extent of unitary equivalence-operators, about which a good deal more is known, of the same structure as $A$ but with $k(x, t)$ of the special form $\phi(x) \bar{\phi}(t)$.

Let $E$ be a set of real numbers of positive measure and suppose that that

(1.1) $h(x)$ is essentially bounded, real and measurable on $E$ and

(1.2) $k(x, t)$ is essentially bounded, measurable and satisfies $k(x, t)=$ $\bar{k}(t, x)$ on $E \times E$. Then the transformation $f \rightarrow A f$, where

$$
(A f)(x)=h(x) f(x)+i \int_{E} k(x, t)(x-t)^{-1} f(t) d t,
$$

the integral being interpreted as a Cauchy principal value, is a bounded, self-adjoint operator on $L^{2}(E)$. Concerning such integrals, see Muskhelishvili [4], also Calderón [1], Putnam [7, 8], Schwartz [13, 14]. The spectral theory of $A$ when $k(x, t)$ is of the form

$$
k(x, t)=\phi(x) \bar{\phi}(t),
$$

where

(1.5) $\phi(x)$ is essentially bounded and measurable on $E$, has been extensively investigated. See, in particular, Koppelman [3], Pincus $[5,6]$, and Rosenblum [11], the latter containing a treatment of the theory for $E$ arbitrary.

Let $K$ denoted the self-adjoint integral operator defined on $L^{2}(E)$ by

$$
(K f)(x)=\int_{E} k(x, t) f(t) d t, \quad f \in D_{K} \subset L^{2}(E) .
$$

An important property of $K$ which will be studied in this paper is that of positivity, $K \geqq 0$, that is

$$
(K f, f) \geqq 0 \quad \text { for } f \in D_{K} \text {. }
$$

It is seen that operators $K$ with kernels $k(x, t)$ given by (1.4) satisfy 
(1.7). It will be shown in this paper that for certain $K$ satisfying (1.7), in many respects, the operator $A$ of (1.3) is similar to a corresponding operator in which $k(x, t)$ has the special form (1.4). It will be convenient to recall first a few of the known properties of the singular integral operators in question.

If $B$ is defined by

$$
(B f)(x)=h(x) f(x)+i \int_{E} \phi(x) \bar{\phi}(t)(x-t)^{-1} f(t) d t
$$

for $f \in L^{2}(E)$, and if

$$
\phi(x) \neq 0 \quad \text { a.e. on } E
$$

and if

$$
(-\infty, \infty)-E \text { has positive measure },
$$

it is known that $B$ is absolutely continuous. (Recall that a self-adjoint operator $H$ with spectral resolution $H=\int \lambda d E_{\lambda}$ is said to be absolutely continuous if $\left\|E_{\lambda} f\right\|^{2}$ is an absolutely continuous function of $\lambda$ for all $f$ in the underlying Hilbert space.) For the general case, in which $E$ is arbitrary, see Rosenblum [11]. If $E$ is assumed to satisfy, instead of (1.10), the stronger relation

$$
E \cap J \quad \text { has measure } 0
$$

for some open interval $J$, the absolute continuity of $B$ was established, by means of commutator theory, in Putnam [7]. More generally, it was shown there that if $h(x)$ and $k(x, t)$ satisfy (1.1), (1.2) and (1.7) and if, in addition,

$$
k(x, t)=0 \text { a.e. on } E \times F \text { implies } F \text { has measure } 0,
$$

where $F$ is any measurable subset of $E$, and if, finally, (1.11) holds, then $A$ of (1.3) is absolutely continuous. Whether this last result holds for such a function $k(x, t)$ if (1.11) is weakened to (1.10) (as is the case if $k(x, t)$ is of the special form (1.4)) is apparently not known.

There will be proved the following:

THEOREM. Let $E$ be a compact set and suppose that $h(x)$ satisfies (1.1) and that $k(x, t)$ is of class $C^{1}$ on $E \times E$ (that is, on some closed rectangle containing $E \times E$ ) and satisfies (1.2). In addition, suppose that

$$
k(x, x)>0 \quad \text { a.e. on } E .
$$

Define the bounded, self-adjoint integral operator $A_{k}$ on $L^{2}(E)$ by

$$
\left(A_{k} f\right)(x)=h(x) f(x)+i \int_{E} k^{\frac{1}{2}}(x, x) k^{\frac{1}{2}}(t, t)(x-t)^{-1} f(t) d t .
$$


Then $A_{k}$ is absolutely continuous and $A$ of (1.3) satisfies

$$
A=A_{k}+M,
$$

where $M$ is a Hilbert-Schmidt operator (hence, in particular, the essential spectrum of $A$ and the spectrum of $A_{k}$ coincide). If, in addition, it is assumed that (1.7) holds, then $A$ is also absolutely continuous.

As a consequence of the above results and the Rosenblum-Kato perturbation theory (Kato [2], Rosenblum [10]; see, e.g., Putnam [8]) one has the following:

CoROLLARY. Let $E$ be a compact set and suppose that $h(x)$ satisfies (1.1) and that $k(x, t)$ is of class $C^{1}$ on $E \times E$ and satisfies (1.2), (1.7) and (1.13). If $M$ of (1.15) is of trace class then $A$ is unitarily equivalent to $A_{k}$, so that

$$
A=U A_{k} U^{*} \quad \text { for some unitary operator } U \text {. }
$$

REMARK. It may be noted that if (1.7) holds then necessarily $k(x, x) \geqq 0$ a.e. on $E$ ( $k$ being continuous), so that the operator $A_{k}$ of (1.14) is certainly defined in this case. Of course, the relation $k(x, x) \geqq 0$ a.e., or even (1.13), may hold even though (1.7) does not.

The theorem will be proved in $\S 2$. Some lemmas will be given in $\S 3$ and an example discussed in $\S 4$. Finally, a few applications of the results to operators $A$ of (1.3) when $k(x, t)$ is of the form

$$
k(x, t)=[a(x)-a(t)](x-t)^{-1}
$$

will be given in $\S 5$.

2. Proof of theorem. It will first be shown that

$$
k(x, t)=k^{\frac{1}{2}}(x, x) k^{\frac{1}{2}}(t, t)+m(x, t)(x-t),
$$

where $m(x, t)$ is bounded on $E$. To this end, note that in view of the $C^{1}$ hypothesis on $k(x, t)$, one has $k(x, t)=k(x, x)+0_{x}(1)(x-t)$, where $0_{x}(1)$ denotes here and below a factor which is bounded as $t \rightarrow x$ $(x$, fixed). Suppose first that $k(x, x)>0$ ( $x$ fixed). Then

$$
k^{\frac{1}{2}}(x, x)=k^{\frac{1}{2}}(t, t)+0_{x}(1)(x-t),
$$

again by the $C^{1}$ hypothesis on $k(x, t)$ and, consequently,

$$
k(x, t)=k^{\frac{1}{2}}(x, x) k^{\frac{1}{2}}(t, t)+0_{x}(1)(x-t) .
$$

If $k(x, x)=0$ however, it is clear that this last relation still remains 
valid. Since $E$ is compact, it is clear that the $O_{x}(1)$ factor can be replaced by a factor $m(x, t)$ bounded on $E$ and (2.1) then follows.

According to the definitions of $A, A_{k}$ in (1.3) and (1.14), one has (1.15), where the bounded, self-adjoint operator $M$ is given by

$$
(M f)(x)=i \int_{E} m(x, t) f(t) d t,
$$

and $m(x, t)$ is defined in (2.1). In view of (1.13) (cf. (1.9)), then, as noted above, when $k(x, t)$ has the form (1.4), $A_{k}$ is absolutely continuous. Since $m(x, t)$ is bounded and $E$ has finite measure, it is clear that $M$ is a Hilbert-Schmidt operator.

Since $k(x, t)$ is continuous on $E$, relation (1.12) is a consequence of (1.13). Since (1.11) surely holds in the present case, then, as noted earlier, relation (1.7) implies the absolute continuity of $A$ in (1.3). This completes the proof of the Theorem.

REMARKS. Both the theorem and its corollary permit certain obvious generalizations in which the smoothness and compactness hypotheses on $k(x, t)$ and $E$ respectively may be relaxed. For instance, suppose that $E$ satisfies (1.11), that (1.1) and (1.2) hold and also that $k(x, t)$ is continuous on $E \times E$. (The continuity assumption assures in particular that the evaluation of $k(x, t)$ on the diagonal $x=t, x$ in $E$, is at least meaningful, as contrasted with the situation when, say, $k(x, t)$ is only measurable.) Suppose in addition that (2.1) holds with

$$
m(x, t) \in L^{2}(E \times E) .
$$

It is seen that with these modified hypotheses, along with the earlier one (1.3) and, in the latter part of the Theorem, also (1.7), generalizations of the theorem, and corollary are easily formulated.

3. Some lemmas. For later use it will be convenient to have the following lemmas:

LEMMA 1. Let $b(x)$ be measurable and satisfy $b(x) \geqq$ const. $>0$ on $E$. Then the operator $K$ of (1.6) with kernel

$$
k(x, t)=[b(x)+b(t)]^{-1}
$$

satisfies (1.7).

Proof. It is clear that $e^{-[b(x)+b(t)] \lambda}$ is bounded and of the type (1.4) for every $\lambda \geqq 0$ and hence $\int_{0}^{\infty} e^{-[b(x)+b(t)] \lambda} d \lambda=[b(x)+b(t)]^{-1}$ yields a kernel for which (1.7) holds. 
LEMMA 2. Let $E$ be a compact set and suppose that $k(x, t)$ and $j(x, t)$ are continuous kernel functions on $E \times E$ for which the corresponding integral operators satisfy (1.7). Then the product $n(x, t)=$ $k(x, t) j(x, t)$ is also a kernel for which the corresponding integral operator satisfies (1.7).

Proof. One has

$$
k(x, t)=\sum \lambda_{n} \phi_{n}(x) \bar{\phi}_{n}(t) \quad \text { and } \quad j(x, t)=\sum \mu_{m} \psi_{m}(x) \bar{\psi}_{m}(t),
$$

where the $\phi$ 's and $\psi$ 's are continuous, $\lambda_{n}, \mu_{m} \geqq 0$, and where the series of (3.2) converge uniformly, even absolutely uniformly on $E \times E$; cf. Riesz and Sz.-Nagy [9, p. 245]. Hence $n(x, t)=\sum \sum \lambda_{n} \mu_{m} \xi_{n m}(x) \bar{\xi}_{n m}(t)$, where $\xi_{n m}(x)=\phi_{n}(x) \psi_{m}(x)$, has similar properties. The nonnegative property of the integral operator with kernel $n(x, t)$ follows from $\lambda_{n}, \mu_{m} \geqq 0$ and the separable structure of the above double series.

LEMMA 3. Let $E$ be a compact set and suppose that $k(x, t)$ is continuous on $E \times E$ and is the kernel of an integral operator $K$ satisfying (1.7). Then

$$
\int_{E} k(x, x) d x=\sum \lambda_{n},
$$

where the $\lambda_{n}$ 's are the eigenvalues of $K$.

Proof. This fact is well-known; cf. Riesz and Sz.-Nagy [9, p. 245].

4. An example. Let $k(x, t)$ be defined by

$$
k(x, t)=(x+t)^{-1}
$$

on $E \times E$, where $E$ is a compact subset of $(0, \infty)$. It follows from Lemma 1 that the associated integral operator $K$ satisfies (1.7). Since $k(x, x)=1 / 2 x$, then $m(x, t)$ of $(2.1)$ satisfies

$$
m(x, t)=-\frac{1}{2}\left(t^{-\frac{1}{2}}-x^{-\frac{1}{2}}\right)\left(x^{\frac{1}{2}}+t^{\frac{1}{2}}\right)^{-1}(x+t)^{-1} .
$$

If $h(x, t)=\left(x^{\frac{1}{2}}+t^{\frac{1}{2}}\right)^{-1}(x+t)^{-1}$ then relation (4.2) states that the operator $M$ of (2.2) is of the form

$$
M=\frac{1}{2} i\left(x^{-\frac{1}{2}} H-H x^{-\frac{1}{2}}\right), \quad \text { where }(H f)(x)=\int_{E} h(x, t) f(t) d t .
$$

Now $H \geqq 0$ (Lemmas 1,2$)$ and $\int_{E} h(x, x) d x<\infty$; hence, by Lemma 3 , $H$ is of trace class. Since the product of a bounded operator and a 
trace class operator as well as the sum of trace class operators all belong to this class (cf. Schatten [12]) it follows from (4.3) that $M$ is of trace class. Consequently, (1.16) holds in this case.

5. Differential quotient kernels. Suppose that $k(x, t)$ has the special form (1.17) where $a(x)$ is of class $C^{2}$ on the compact set $E$. Then it is clear that $k(x, t)$, where $k(x, x)=a^{\prime}(x)$, is of class $C^{1}$ on $E \times E$. (For a similar integral, see Calderón [1, p. 435].) In case it is also assumed that

$$
a^{\prime}(x)>0 \text { a.e. on } E,
$$

then the theorem of $\S 1$ is applicable. The operator $A_{k}$ of (1.14) now becomes

$$
\left(A_{a^{\prime}} f\right)(x)=h(x) f(x)+i \int_{E}\left[a^{\prime}(x) a^{\prime}(t)\right]^{\frac{1}{2}}(x-t)^{-1} f(t) d t
$$

and, by (1.15),

$$
A=A_{a^{\prime}}+M \quad(k \text { given by }(1.17)) .
$$

In case $a(x) \equiv x$ then $k(x, t) \equiv 1$ and the integral operator portion of $A$ reduces to a constant multiple of the Hilbert transform. In particular, the kernel satisfies (1.7) and also $A=A_{k}$ in this case. It is not so apparent however just what other functions $a(x)$ will yield kernels $k(x, t)$ of the type (1.17) satisfying (1.7). (It is clear though that if $a(x) \neq 0$ has this property then so does $-1 / a(x))$. Some examples will be considered below.

It will be convenient to suppose from now on that $E$ is a compact subset of $(0, \infty)$.

That (5.1) is not sufficient to guarantee that $k$ satisfy $(1.7)$ is clear if one chooses

$$
a(x)=x^{2} .
$$

A simple direct verification shows that $K$ of (1.6), with $k$ defined by (1.17) and (5.4), fails to satisfy (1.7). On the other hand, it is seen that (1.13) holds and hence $A_{a^{\prime}}$ of (5.2) is absolutely continuous. Furthermore, the operator $M$ of (1.15) belongs to trace class. In fact, $m(x, t)$ of $(2.2)$ is now given by $m(x, t)=\left(x^{\frac{1}{2}}-t^{\frac{1}{2}}\right)\left(x^{\frac{1}{2}}+t^{\frac{1}{2}}\right)^{-1}$. Hence, by an argument like that of $\S 4$, the result follows.

Although it has not been shown that $A$ of (1.3), with $k(x, t)$ defined by (1.17) and (5.4), is absolutely continuous, nevertheless it does follow from the Rosenblum-Kato theory that, in view of relation (5.3), the fact that $A_{a^{\prime}}$ is absolutely continuous, and that $M$ is of trace class, that $A$ has an absolutely continuous part which is unitarily 
equivalent to $A_{a^{\prime}}$. Whether $A$ itself is absolutely continuous will remain undecided however.

In case $a(x)$ is given by

$$
a(x)=x^{\frac{1}{2}}
$$

though, then the integral operator $K$ of (1.6) with $k(x, t)$ defined by (1.17) and (5.5) satisfies (1.7). In fact, $k(x, t)=\left(x^{\frac{1}{2}}-t^{\frac{1}{2}}\right)(x-t)^{-1}=$ $\left(x^{\frac{1}{2}}+t^{\frac{1}{2}}\right)^{-1}$ and the assertion follows from Lemma 1. Again, it is easily shown that the operator $M$ of (5.3) belongs to trace class. For now,

$$
m(x, t)=-\frac{1}{2}\left(t^{-\frac{1}{4}}-x^{-\frac{1}{4}}\right)\left[\left(x^{\frac{1}{2}}+t^{\frac{1}{2}}\right)^{-2}\left(x^{\frac{1}{4}}+t^{\frac{1}{4}}\right)^{-1}\right] .
$$

Since the multiplication operator $x^{-\frac{1}{4}}$ is bounded on $L^{2}(E)$ and since $[\cdots]$ is the kernel of an operator satisfying (1.7) (Lemmas 1,2) and which belongs to trace class (Lemma 3 ), it follows, as in $\S 4$, that $M$ is of trace class. Thus, in this case, $A$ of (1.3) is unitarily equivalent to $A_{a^{\prime}}$ of (5.2).

It follows from Lemmas 1, 2 that any function of the form

$$
k_{n}(x, t)=\left(x^{1 / 2^{n}}-t^{1 / 2^{n}}\right)(x-t)^{-1}
$$

is the kernel of an operator $K$ satisfying (1.7). (This can be seen by factoring $(x-t)$.) Since

$$
2^{n}\left(x^{1 / 2^{n}}-t^{1 / 2^{n}}\right)=\left(x^{1 / 2^{n}}-1\right) / 2^{-n}-\left(t^{1 / 2^{n}}-1\right) / 2^{-n}
$$

tends to $\log x-\log t$ as $n \rightarrow \infty$, it is clear that

$$
k(x, t)=(\log x-\log t)(x-t)^{-1}
$$

is also a kernel for which $K$ satisfies (1.7). Thus, $k(x, t)$ of (1.17) is such that $K$ satisfies (1.7) whenever $a(x)=x^{1 / 2^{n}}(n=0,1,2, \cdots)$ or $a(x)=\log x$. Use of the lemmas can produce other examples of functions $a(x)$ for which $k(x, \mathrm{t})$ of $(1.17)$ is the kernel of an operator $K$ satisfying (1.7). Thus, if $E$ is now a compact subset of $(1, \infty)$, then (Lemmas 1,2), the product of $k(x, t)$ of $(5.7)$ and $\left((\log x)^{\frac{1}{2}}+(\log t)^{\frac{1}{2}}\right)^{-1}$, that is, $\left((\log x)^{\frac{1}{2}}-(\log t)^{\frac{1}{2}}\right)(x-t)^{-1}$, is also such a kernel. An argument like that used above shows that $a(x)=\log (\log x)$ is another such kernel. Similarly, $a(x)=\log (\log (\log x)), \cdots$, also serve, with appropriate restrictions on the set $E$.

In view of the possible applications of the Theorem, especially if $k(x, t)$ is such that $K$ satisfies (1.7), it would be useful to have some general criterion assuring that a kernel $k(x, t)$ of the type (1.17) is one for which (1.7) holds. 


\section{REFERENCES}

1. A. P. Calderón, Singular integrals, Bull. Amer. Math. Soc. 72 (1966), 427-465.

2. T. Kato, Perturbation of continuous spectra by trace class operators, Proc. Japan Acad. 33 (1957), 260-264.

3. W. Koppelman, Spectral multiplicity theory for a class of singular integral operators, Trans. Amer. Math. Soc. 113 (1964), 87-100.

4. N. J. Muskhelishvili, Singular Integral Equations, Groningen, Noordhoff, 1953.

5. J. D. Pincus, On the spectral theory of singular integral operators, Trans. Amer. Math. Soc. 113 (1964), 101-128.

6. - Commutators, generalized eigenfunction expansions and singular integral operators, Trans. Amer. Math. Soc. 121 (1966), 358-377.

7. C. R. Putnam, The spectra of generalized Hilbert transforms, J. Math. and Mech. 14 (1965), 857-872.

8. - Commutation properties of Hilbert space operators and related topics, Frgebnisse der Math. 36 (1967).

9. F. Riesz and B. Sz.-Nagy, Functional Analysis, Ungar Pub. Co., 1955.

10. M. Rosenblum, Perturbation of the continuous spectrum and unitary equivalence, Pacific J. Math. 7 (1957), 997-1010.

11. , A spectral theory for self-adjoint singular integral operators, Amer. J' Math. 88 (1966), 314-328.

12. R. Schatten, A theory of cross spaces, Ann. Math. Studies, 1950.

13. J. Schwartz, Some non-selfadjoint operators, Comm. Pure Appl. Math. 13 (1960), 609-639.

14. - Some results on the spectra and spectral resolutions of a class of singular integral operators, Comm. Pure Appl. Math. 15 (1962), 75-90.

Received June 22, 1967. This work was supported by a National Science Foundation research grant.

PuRdue University 


\section{PACIFIC JOURNAL OF MATHEMATICS}

\section{EDITORS}

\section{H. ROYDEN}

Stanford University

Stanford, California

\author{
R. R. Phelps \\ University of Washington \\ Seattle, Washington 98105
}

\section{J. DugundJI}

Department of Mathematics University of Southern California Los Angeles, California 90007

\section{RICHARD ARENS}

University of California

Los Angeles, California 90024

\section{ASSOCIATE EDITORS}

\section{E. F. BECKENBACH}

B. H. NEUMANN

F. WOLF

K. YOSIDA

\section{SUPPORTING INSTITUTIONS}

UNIVERSITY OF BRITISH COLUMBIA CALIFORNIA INSTITUTE OF TECHNOLOGY UNIVERSITY OF CALIFORNIA MONTANA STATE UNIVERSITY UNIVERSITY OF NEVADA NEW MEXICO STATE UNIVERSITY OREGON STATE UNIVERSITY UNIVERSITY OF OREGON OSAKA UNIVERSITY UNIVERSITY OF SOUTHERN CALIFORNIA

\author{
STANFORD UNIVERSITY \\ UNIVERSITY OF TOKYO \\ UNIVERSITY OF UTAH \\ WASHINGTON STATE UNIVERSITY \\ UNIVERSITY OF WASHINGTON \\ AMERICAN MATHEMATICAL SOCIETY \\ CHEVRON RESEARCH CORPORATION \\ TRW SYSTEMS
}

NAVAL WEAPONS CENTER

Mathematical papers intended for publication in the Pacific Journal of Mathematics should be in typed form or offset-reproduced, double spaced with large margins. Underline Greek letters in red, German in green, and script in blue. The first paragraph or two must be capable of being used separately as a synopsis of the entire paper. It should not contain references to the bibliography. Manuscripts, in duplicate if possible, may be sent to any one of the four editors. All other communications to the editors should be addressed to the managing editor, Richard Arens, University of California, Los Angeles, California 90024.

Each author of each article receives 50 reprints free of charge; additional copies may be obtained at cost in multiples of 50 .

The Pacific Journal of Mathematics is published monthly. Effective with Volume 16 the price per volume (3 numbers) is $\$ 8.00$; single issues, $\$ 3.00$. Special price for current issues to individual faculty members of supporting institutions and to individual members of the American Mathematical Society: $\$ 4.00$ per volume; single issues $\$ 1.50$. Back numbers are available.

Subscriptions, orders for back numbers, and changes of address should be sent to Pacific Journal of Mathematics, 103 Highland Boulevard, Berkeley 8, California.

Printed at Kokusai Bunken Insatsusha (International Academic Printing Co., Ltd.), 7-17, Fujimi 2-chome, Chiyoda-ku, Tokyo, Japan.

PUBLISHED BY PACIFIC JOURNAL OF MATHEMATICS, A NON-PROFIT CORPORATION

The Supporting Institutions listed above contribute to the cost of publication of this Journal, but they are not owners of publishers and have no responsibility for its content or policies. 


\section{Pacific Journal of Mathematics}

\section{Vol. 27, No. $2 \quad$ February, 1968}

Leonard E. Baum and George Roger Sell, Growth transformations for

functions on manifolds ............................ 211

Henry Gilbert Bray, A note on CLT groups ................... 229

Paul Robert Chernoff, Richard Anthony Rasala and William Charles

Waterhouse, The Stone-Weierstrass theorem for valuable fields....... 233

Douglas Napier Clark, On matrices associated with generalized

interpolation problems ................................

Richard Brian Darst and Euline Irwin Green, On a Radon-Nikodym theorem for finitely additive set functions . ...................... 255

Carl Louis DeVito, A note on Eberlein's theorem..................... 261

P. H. Doyle, III and John Gilbert Hocking, Proving that wild cells exist . . . 265

Leslie C. Glaser, Uncountably many almost polyhedral wild $(k-2)$-cells in

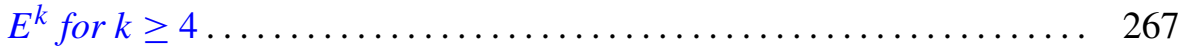

Samuel Irving Goldberg, Totally geodesic hypersurfaces of Kaehler

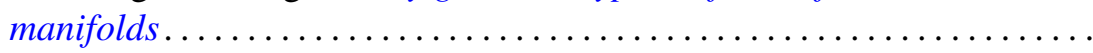

Donald Goldsmith, On the multiplicative properties of arithmetic functions .................................... 283

Jack D. Gray, Local analytic extensions of the resolvent ............ 305

Eugene Carlyle Johnsen, David Lewis Outcalt and Adil Mohamed Yaqub,

Commutativity theorems for nonassociative rings with a finite division ring homomorphic image ....................

André (Piotrowsky) De Korvin, Normal expectations in von Neumann

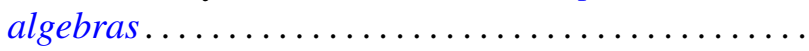

James Donald Kuelbs, A linear transformation theorem for analytic

Feynman integrals..........................

W. Kuich, Quasi-block-stochastic matrices ................... 353

Richard G. Levin, On commutative, nonpotent archimedean

semigroups ............................... 365

James R. McLaughlin, Functions represented by Rademacher series ... . . . 373

Calvin R. Putnam, Singular integrals and positive kernels............ 379

Harold G. Rutherford, II, Characterizing primes in some noncommutative

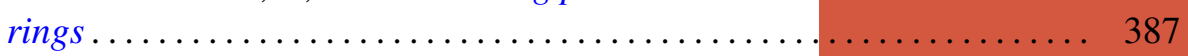

Benjamin L. Schwartz, On interchange graphs................... 393

Satish Shirali, On the Jordan structure of complex Banach *algebras . . . . . 397

Earl J. Taft, A counter-example to a fixed point conjecture............. 405

J. Roger Teller, On abelian pseudo lattice ordered groups ..... 